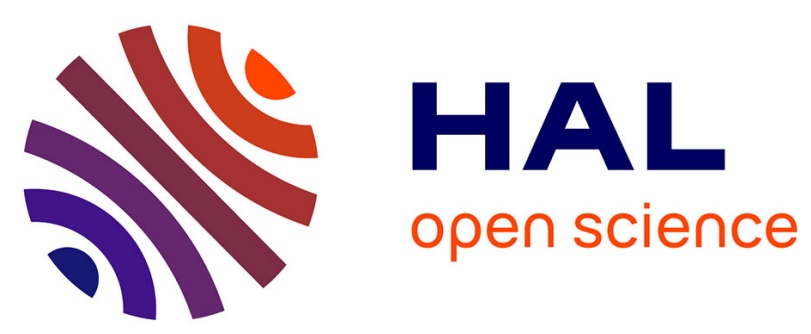

\title{
Mémoire émotionnelle du soi
}

C. Lemogne, L. Bergouignan, P. Fossati

\section{To cite this version:}

C. Lemogne, L. Bergouignan, P. Fossati. Mémoire émotionnelle du soi. Annales MédicoPsychologiques, Revue Psychiatrique, 2011, 169 (2), pp.116. 10.1016/j.amp.2010.12.004 . hal00727594

\section{HAL Id: hal-00727594 \\ https://hal.science/hal-00727594}

Submitted on 4 Sep 2012

HAL is a multi-disciplinary open access archive for the deposit and dissemination of scientific research documents, whether they are published or not. The documents may come from teaching and research institutions in France or abroad, or from public or private research centers.
L'archive ouverte pluridisciplinaire HAL, est destinée au dépôt et à la diffusion de documents scientifiques de niveau recherche, publiés ou non, émanant des établissements d'enseignement et de recherche français ou étrangers, des laboratoires publics ou privés. 


\section{Accepted Manuscript}

Title: Mémoire émotionnelle du soi

Authors: C. Lemogne, L. Bergouignan, P. Fossati

PII: $\quad$ S0003-4487(10)00410-5

DOI: $\quad$ doi:10.1016/j.amp.2010.12.004

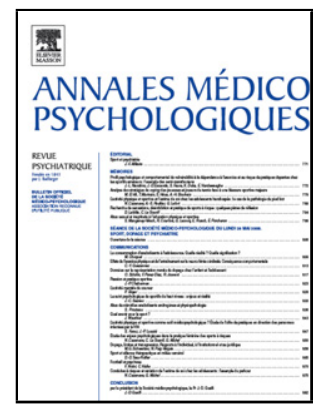

Reference: $\quad$ AMEPSY 1282

To appear in: $\quad$ Annales Médico-Psychologiques

Please cite this article as: Lemogne C, Bergouignan L, Fossati P, Mémoire émotionnelle du soi, Annales medio-psychologiques (2010), doi:10.1016/j.amp.2010.12.004

This is a PDF file of an unedited manuscript that has been accepted for publication. As a service to our customers we are providing this early version of the manuscript. The manuscript will undergo copyediting, typesetting, and review of the resulting proof before it is published in its final form. Please note that during the production process errors may be discovered which could affect the content, and all legal disclaimers that apply to the journal pertain. 


\section{Communication}

Mémoire émotionnelle du soi

Emotional memory of the self

C. Lemogne $^{\text {a,b,c }}$, L. Bergouignan ${ }^{\text {d }}$, P. Fossati ${ }^{\text {a,e,f }}$

${ }^{a}$ CNRS, USR 3246, Paris, France

${ }^{b}$ Université Paris Descartes, Paris, France

${ }^{c}$ Assistance Publique-Hôpitaux de Paris, Service de psychologie clinique et psychiatrie de liaison, Hôpital Européen Georges Pompidou, Paris, France

${ }^{d}$ Karolinska Institute, Department of Neuroscience, Stockholm, Suède

${ }^{e}$ Université Pierre et Marie Curie, Paris, France

${ }^{f}$ Assistance Publique-Hôpitaux de Paris, Service de psychiatrie de l'adulte, Hôpital PitiéSalpêtrière, Paris, France

Auteur correspondant : Dr Cédric Lemogne, Service de psychologie clinique et psychiatrie de liaison, Hôpital Européen Georges Pompidou, 20, rue Leblanc, 75015 Paris, France

Tél. : 0156093371

Fax : 0156093146

Adresse email : cedric.lemogne@ egp.aphp.fr

\section{Résumé}

Les émotions structurent la mémoire autobiographique (MA) en distinguant les événements importants pour le soi narratif, c'est-à-dire pour la poursuite de buts à long terme. Le rappel en MA est un processus de reconstruction qui fournit au soi une trame narrative cohérente dans le temps à l'origine d'un sentiment d'identité. Alors que le cortex préfrontal sous-tend les processus de reconstruction et de référence à soi, respectivement par ses régions latérales et médiales, le cortex temporal sous-tend les aspects sémantiques et épisodiques du rappel en MA, respectivement par ses régions latérales et médiales. De plus, les structures corticales médianes postérieures et l'amygdale sont impliquées dans les aspects visuels et émotionnels du souvenir. Toutes ces régions ont un fonctionnement anormal dans la dépression majeure, qui, sans surprise, est associé à des troubles de la MA combinant congruence à l'humeur, surgénéralisation, souvenirs intrusifs et perspective visuelle à la troisième personne. 
Mots clés : Cortex préfrontal ; Dépression ; Émotion ; Mémoire autobiographique ; Soi

\begin{abstract}
Emotion shapes autobiographical memory (AM) by tagging events that are relevant for the narrative self, which may be viewed as a hierarchical network of interconnected goals. Subsequent AM retrieval is a reconstruction process that grounds the self by providing coherent narratives organized to elicit a sense of identity across the time. The lateral and medial prefrontal cortices underlie reconstruction and self-referential processing, respectively, whereas the lateral and medial temporal cortices underlie semantic and episodic aspects. Additionally the posterior cortical midline structures and the amygdala are involved in visual and emotional aspects, respectively. These regions have been found to display aberrant functioning in major depression. Accordingly, major depression is associated with impairments of AM retrieval combining mood-congruency, overgenerality, intrusive memories and third-person visual perspective.
\end{abstract}

Keywords: Autobiographical memory; Depression; Emotion; Prefrontal cortex; Self

\title{
1. Introduction
}

La notion de soi renvoie à deux concepts principaux, le soi minimal et le soi narratif. Le premier correspond à la conscience de soi en tant que sujet de l'expérience immédiate, et le second à la représentation de soi en tant qu'objet de connaissance étendu dans le temps. Il existe un relatif consensus en philosophie de l'esprit autour d'une vision constructiviste du soi narratif [1]. Le soi narratif serait une fiction autogénérée destinée à donner un sens personnel à une succession d'expériences du soi minimal. Les émotions jouent un rôle fondamental dans le devenir mnésique de ces expériences qui pourront, le cas échéant, influencer le comportement ultérieur du sujet via sa mémoire autobiographique (MA) [4]. Le soi narratif peut donc être considéré comme une «fiction opérante ». Le cas de la dépression illustrera ici le rôle de nos émotions dans la construction de cette fiction en psychopathologie.

\section{Aspects normaux}

\subsection{Le modèle de Conway}


Selon Conway [4], les différents systèmes de mémoire peuvent être hiérarchisés en fonction de l'échéance des buts dont ils sous-tendent la poursuite. La mémoire de travail soustendrait la poursuite de buts à très court terme : effectuer un calcul, suivre une conversation, réaliser une séquence d'actions, etc. La mémoire épisodique serait impliquée dans la poursuite de buts à moyen terme, c'est-à-dire à l'échelle d'une journée. Elle permettrait d'enregistrer la trace des actions déjà effectuées ce jour afin d'organiser les actions à venir (mémoire prospective). À long terme, la majorité des actions accomplies pendant la journée sont rapidement oubliées. En revanche, si une des actions accomplies est liée à la poursuite d'un but à long terme, la probabilité de consolidation du souvenir augmente considérablement en fonction de l'intensité de l'émotion ressentie. La MA permettrait ainsi la reconstruction de souvenirs épisodiques attestant de la réalisation (ou non) de buts à long terme, c'est-à-dire à l'échelle d'une vie. Cette reconstruction du souvenir consisterait à mettre en relation des éléments autobiographiques appartenant à trois niveaux de spécificité croissante : connaissances associées à une période de vie, à un événement général, et des détails perceptivo-sensoriels spécifiques d'un événement. Cette reconstruction ferait appel à un cycle de récupération sous la dépendance d'un système exécutif central, dénommé soi opérant (working self), supposé rendre compte non seulement du rôle de la mémoire de travail dans la reviviscence d'un souvenir mais également de l'influence du soi narratif constitué sur la MA.

\subsection{Bases cérébrales du rappel en MA}

Pour des raisons évidentes, l'étude des bases cérébrales de la MA par l'imagerie cérébrale fonctionnelle est essentiellement confinée à celles du rappel [15]. Alors que la partie latérale du cortex préfrontal sous-tendrait la dimension de reconstruction, la partie médiane (cortex médian préfrontal, CMPF) sous-tendrait la validation de l'expérience subjective ainsi reconstruite comme appartenant au soi. Plus généralement, le CMPF appartient à un réseau de régions cérébrales incluant également le cortex cingulaire postérieur, le précunéus, le cortex rétrosplénial et la jonction temporo-pariétale. Ces régions ont la particularité d'être plus actives «au repos » ou lors de tâches impliquant le traitement d'informations autogénérées que lors de tâches impliquant le traitement d'informations externes [6], d'où la notion de réseau de fonctionnement cérébral par défaut. La plus grande activation ou la moindre désactivation de ce réseau lors de tâches de référence à soi sont généralement interprétées comme témoignant de l'implication de ce type de processus dans le fonctionnement cérébral au repos. 
En ce qui concerne le lobe temporal, les régions latérales seraient impliquées dans les aspects sémantiques de la reconstruction du souvenir (informations associées à une période de vie ou à un type d'événement général), alors que les régions médiales, en particulier l'hippocampe, seraient impliquées dans les aspects épisodiques, c'est-à-dire la contextualisation du souvenir permettant sa reviviscence et l'accès à un niveau de conscience autonoétique. Le concept de conscience autonoétique renvoie à la capacité de voyager mentalement dans le passé pour revivre une expérience subjective du soi [4]. Ce voyage dans le temps n'est cependant pas limité au passé et comprend également la capacité d'imaginer le futur, ou prospection. De fait, les bases cérébrales du rappel en MA et de la prospection sont qualitativement similaires. Enfin, en avant de l'hippocampe, l'amygdale confère un avantage mnésique aux événements émotionnels [7] et sous-tend donc le rôle structurant des émotions dans la MA [4].

\section{Aspects pathologiques : exemple de la dépression}

\subsection{MA et dépression}

De nombreuses études montrent que les patients déprimés produisent des souvenirs peu spécifiques en réponse à des indices de rappel $[12,16]$. Le rappel autobiographique des patients déprimés s'expliquerait surtout par un excès de souvenirs catégoriels, c'est-à-dire se référant à des événements survenus plus d'une fois. Ce phénomène, dit de surgénéralisation, prédirait une mauvaise évolution à court terme de l'épisode dépressif, un risque de suicide plus élevé, et serait associé à une diminution de la capacité de résolution de problèmes. Selon Williams et al. [16], trois facteurs essentiels contribueraient à la surgénéralisation dans la dépression :

1) des processus de capture des capacités cognitives et de rumination ;

2) un évitement fonctionnel de l'accès au souvenir spécifique ;

3) les troubles des fonctions exécutives.

Plus récemment, notre équipe s'est intéressée à une mesure indirecte de la focalisation sur soi au cours du rappel de souvenirs autobiographiques. La MA, qui repose sur des bases cérébrales en grande partie communes à celles de la référence à soi (cf. supra) subit les contraintes de continuité et de cohérence du soi narratif. La perception d'une discontinuité entre le soi actuel et le soi évoqué lors du rappel influence la reconstruction ou non d'une 
expérience visuelle analogue à celle de l'encodage. Cette expérience visuelle peut notamment adopter deux points de vue [14] : le point de vue d'acteur, avec lequel la scène est revue de l'intérieur comme lors de l'événement ; le point de vue de spectateur, avec lequel la scène et le sujet sont revus de l'extérieur, comme dans un film. Par rapport à des sujets sains, les patients déprimés ont tendance à reconstruire leurs souvenirs positifs selon un point de vue de spectateur [11], y compris après rémission complète [3].

Sur la base de nombreuses études corrélationnelles et expérimentales en psychologie sociale, l'adoption d'un point de vue de spectateur lors du rappel en MA est généralement interprétée comme témoignant d'une focalisation sur soi accrue [14]. Lors du rappel en MA, le soi opérant comparerait le comportement passé du sujet avec celui qu'il aurait au moment du rappel dans un contexte similaire. L'hypothèse d'un comportement actuel différent entraînerait :

1) l'attribution du comportement passé à un soi ancien ;

2) la reconstruction d'un point de vue de spectateur (focalisation sur soi).

À l'inverse, l'hypothèse d'un comportement actuel identique entraînerait :

1) l'attribution du comportement passé au contexte ancien ;

2) la reconstruction d'un point de vue d'acteur (focalisation sur le contexte).

Selon cette interprétation, les patients déprimés auraient tendance à attribuer à un soi passé leurs souvenirs positifs, d'où une focalisation sur soi plus importante [3,11].

\subsection{Bases cérébrales putatives}

Les bases cérébrales des anomalies du rappel en MA observées chez les patients déprimés ne sont pas connues. Cependant, il est possible de formuler les quatre hypothèses suivantes avec un degré croissant d'incertitude. Premièrement, l'amygdale, hyperactive au cours de la dépression, joue très vraisemblablement un rôle clé dans la congruence à l'humeur des souvenirs rappelés par les patients déprimés. Par exemple, Hamilton et Gotlib [8] ont montré que l'activation préférentielle de l'amygdale droite pour les stimuli négatifs chez les patients déprimés prédisait la meilleure mémorisation de ces stimuli à long terme. Deuxièmement, le cortex préfrontal latéral, impliqué dans l'inhibition des éléments non pertinents lors du rappel mnésique [1] et anormalement sollicité lors des processus de référence à soi chez les patients déprimés [9], pourrait jouer un rôle dans la fréquence des 
souvenirs intrusifs, en combinaison avec l'hyperactivation de l'amygdale. Troisièmement, le CMPF, de par son implication dans la focalisation sur soi des patients déprimés $[9,10]$, pourrait jouer un rôle dans la fréquence du point de vue de spectateur associé à leurs souvenirs. Quatrièmement, le petit volume de l'hippocampe des patients déprimés est une donnée particulièrement robuste [2], mais dont la signification psychopathologique n'est pas connue. L'implication de l'hippocampe dans les aspects épisodiques du rappel en MA suggère fortement qu'il pourrait jouer un rôle dans la surgénéralisation des patients déprimés.

\section{Conclusion}

En tant que marqueurs de la poursuite des buts du sujet, les émotions contribuent à organiser la MA. Les perturbations émotionnelles observées au cours de la dépression sont donc potentiellement responsables des anomalies du rappel en MA des patients déprimés : surgénéralisation, congruence à l'humeur, souvenirs intrusifs, mais aussi adoption d'un point de spectateur témoignant d'une focalisation sur soi accrue. Des études supplémentaires sont nécessaires pour préciser les bases cérébrales de ces anomalies. En l'état actuel des connaissances, celles-ci ne peuvent être qu'extrapolées à partir de connaissances acquises chez le sujet sain.

\section{Conflit d'intérêt : aucun}

\section{Références}

[1] Anderson MC, Ochsner KN, Kuhl B, et al. Neural systems underlying the suppression of unwanted memories. Science 2004;303:232-5.

[2] Bergouignan L, Chupin M, Czechowska Y, Kinkingnéhun S, Lemogne C, le Bastard G, et al. Can Voxel Based Morphometry, manual segmentation and automated segmentation equally detect hippocampal volume differences in acute depression. Neuroimage 2009;45:2937.

[3] Bergouignan L, Lemogne C, Foucher A, Longin E, Vistoli D, Allilaire JF, Fossati P. Field perspective deficit for positive memories characterizes autobiographical memory in euthymic depressed patients. Behav Res Ther 2008;46:322-33.

[4] Conway MA. Sensory-perceptual episodic memory and its context: autobiographical memory. Phil Trans R Soc Lond B 2001;356:1375-84. 
[5] Gallagher S. Philosophical conceptions of the self: implications for cognitive science. Trends Cogn Sci 2000;4:14-21.

[6] Gusnard DA, Akbudak E, Shulman GL, Raichle ME. Medial prefrontal cortex and selfreferential mental activity: relation to a default mode of brain function. Proc Natl Acad Sci USA 2001;98:4259-64.

[7] Hamann S. Cognitive and neural mechanisms of emotional memory. Trends Cogn Sci 2001;5:394-400.

[8] Hamilton JP, Gotlib IH. Neural substrates of increased memory sensitivity for negative stimuli in major depression. Biol Psychiatry 2008;63:1155-62.

[9] Lemogne C, le Bastard G, Mayberg H, Volle E, Bergouignan L, Lehéricy S, et al. In Search of the Depressive Self: Extended Medial Prefrontal Network during Self-Referential Processing in Major Depression. Soc Cogn Affect Neurosci 2009;4:305-12.

[10] Lemogne C, Mayberg H, Bergouignan L, Volle E, Delaveau P, Lehéricy S, Allilaire JF, Fossati P. Self-referential processing and the prefrontal cortex over the course of depression: A pilot study. J Affect Disord 2010;124:196-201.

[11] Lemogne C, Piolino P, Friszer S, Claret A, Girault N, Jouvent R, et al. Episodic autobiographical memory in depression: Specificity, autonoetic consciousness, and selfperspective. Conscious Cogn 2006;15:258-68.

[12] Lemogne C, Piolino P, Jouvent R, Allilaire JF, Fossati P. Mémoire autobiographique épisodique et dépression. L'Encéphale 2006;32:781-88.

[13] Northoff G, Heinzel A, de Greck M, Bermpohl F, Dobrowolny H, Panksepp J. Selfreferential processing in our brain a metaanalysis of imaging studies on the self. Neuroimage 2006;31:440-57.

[14] Sutin AR, Robins RW. When the "I" looks at the "Me": autobiographical memory, visual perspective, and the self. Conscious Cogn 2008;17:1386-97.

[15] Svoboda E, McKinnon MC, Levine B. The functional neuroanatomy of autobiographical memory: a meta-analysis. Neuropsychologia 2006;44:2189-208.

[16] Williams JM, Barnhofer T, Crane C, Hermans D, Raes F, Watkins E, et al. Autobiographical memory specificity and emotional disorder. Psychol Bull 2007;133:122-48.

\section{Discussion}


$\operatorname{Pr}$ M. Laxenaire - Ma question fait intervenir un niveau un peu différent : pourrait-on dire que vos recherches confirment ou infirment les théories psychanalytiques sur «les souvenirs traumatisants »? Je pensais aussi en vous écoutant au syndrome post-traumatique : peut-on l'expliquer à la lumière de vos recherches sur ce que vous décrivez comme mémoire émotionnelle?

Mme V. Nahama - Un article de 2009 de Cabe et al. (paru dans Psychopharmacology 2009; 205: 667-77 : « Neural representation of reward in recovered depressed patients ») fait état de la présence de déficits dans les bases neuronales des circuits de la récompense chez les déprimés guéris. Comment peut-on intégrer les difficultés de la perception du soi selon un paradigme constructiviste avec les modèles de dysfonctionnement et/ou d'anomalies neuroanatomiques (sensibilité différentielle aux circuits de récompense et de punition)? Quel serait leur impact sur la construction de soi ?

Réponse du Rapporteur - D'un point de vue épistémologique, il n'est pas certain que les recherches en neurosciences cognitives dans le domaine de la mémoire autobiographique (MA) puissent réellement confirmer ou infirmer les théories psychanalytiques. Par exemple, les études portant sur les bases cérébrales du refoulement se focalisent sur les processus d'oubli dirigé en faisant abstraction des contenus et des motifs qui sont au cœur des théories psychanalytiques. Cela dit, les modèles cognitifs actuels de la MA, en particulier celui de Conway, s'inscrivent dans une perspective constructiviste qui était aussi celle de Freud : il n'y a pas de souvenirs; il n'y a que des reconstructions. Dans le cas du syndrome posttraumatique, l'amnésie dissociative, souvent caractérisée par une hypermnésie de certains détails perceptivo-sensoriels au détriment du contexte général de l'événement, vient signer l'échec de cette reconstruction, c'est-à-dire de la capacité à intégrer l'événement traumatique dans une trame narrative cohérente. Le traumatisme psychique apparaît donc ici comme une rupture dans le sentiment d'identité du sujet au cours du temps, sentiment d'identité qui entretient des relations réciproques avec la MA.

La notion de reconstruction est subordonnée à celle de construction. Sur le plan cérébral, cela se traduit par une quasi-identité des réseaux neuronaux impliqués dans la MA et dans l'imagination d'événements futurs [3]. Cette quasi-identité nous invite à concevoir la MA comme une variété particulière de projection mentale de soi, en l'occurrence dans le 
passé, mais qui peut tout aussi bien nous permettre d'imaginer notre futur, d'où la notion élargie de voyage mental dans le temps. Cela nous amène à la question des liens unissant la MA et les systèmes de récompense/punition qui guident nos comportements.

Le développement de la MA chez l'Homme suppose que cette capacité a constitué pour notre espèce un avantage dans la sélection naturelle. Parmi les fonctions attribuées à la MA, on peut notamment spéculer sur son rôle dans la prise de décision. La MA pourrait nous permettre d'anticiper les scénarios possibles, de privilégier ponctuellement une évaluation casuistique de la situation (par opposition au recours à des règles inférentielles), de hiérarchiser les différents éléments du contexte, voire de maintenir une vigilance économique (réciprocité des comportements) et épistémique (fiabilité des informations) vis-à-vis de nos partenaires sociaux. Cela dit, aucune de ces fonctions ne repose nécessairement sur la composante épisodique de la MA, c'est-à-dire sur la capacité à voyager dans le temps pour revivre les événements personnellement vécus. Une MA purement sémantique suffirait. Qu'apporte donc la composante épisodique de la MA ?

La notion de prise de décision suppose de choisir entre au moins deux alternatives et donc d'être capable d'attribuer une valeur relative à chacune de ces alternatives. Or la valeur attribuée à ces alternatives par les systèmes de récompense/punition a tendance à être dévaluée en fonction du délai estimé entre la prise de décision et ses conséquences. En d'autres termes, ces systèmes nous inclineraient plutôt à préférer une récompense minime mais immédiate au détriment d'une récompense supérieure mais différée, voire au prix d'une punition importante mais également différée. Ce phénomène connu des économistes comportementaux sous le terme de delay discounting s'observe toutefois plus facilement en laboratoire que dans la vie réelle, sauf dans la clinique de l'impulsivité. Une hypothèse séduisante suggère que la composante épisodique de la MA, et plus généralement le voyage mental dans le temps, nous sert à contrer les effets de ce delay discounting en actualisant les conséquences émotionnelles anticipées de nos choix [1]. A l'appui de cette hypothèse, il a récemment été montré que les réseaux du voyage mental dans le temps interagissaient avec ceux de la récompense pour nous permettre de nous affranchir des stimuli du présent au profit d'une représentation de l'avenir [2]. Sur le plan clinique, cette hypothèse postule un lien direct, mais non clairement démontré, entre impulsivité et fonctionnement de la MA.

\section{Références}

[1] Boyer P. Evolutionary economics of mental time travel? Trends Cogn Sci 2008;12:21924. 
[2] Peters J, Büchel C. Episodic Future Thinking Reduces Reward Delay Discounting through an Enhancement of Prefrontal-Mediotemporal Interactions. Neuron 2010;66:138-48.

[3] Schacter DL, Addis DR, Buckner RL. Remembering the past to imagine the future: The prospective brain. Nat Rev Neurosci 2007;8:657-61. 